\title{
Our spinal anesthesia experience in a pregnant patient with Wolff Parkinson White syndrome
}

\begin{abstract}
Wolff-Parkinson-White syndrome (WPW) is a rare type of preexcitation. This syndrome is characterized by delta waves, wide QRS complex, and short PR interval. In this case report, we aimed to present our experience with spinal anesthesia method which we preferred to avoid the use of multiple drugs in cesarean section of a pregnant women with WPW syndrome.
\end{abstract}

Volume 8 Issue 6 - 2018

\section{Emine Yitkin, Duygu Demiröz Aslan, Ayșe Gül Ferlengez}

Department of Anesthesiology, Istanbul Educational Research Hospital,Turkey

\author{
Correspondence: Duygu Demiröz Aslan, Department of \\ Anesthesiology, Istanbul Educational Research Hospital, Istanbul,
} Turkey, Email drduygademiroz@hotmail.com

Received: July 22, 2018 | Published: November 21, 2018

\section{Introduction}

Wolff-Parkinson-White syndrome (WPW) is a type of ventricular preexcitation with the presence of accessory pathway which connects atria and ventricles to each other in the conduction tissue, and is seen by $0.1 \%$ to $3 \%$ in general population. The syndrome was described for the first time by Wolff, Parkinson and White as a syndrome accompanied by paroxysmal tachycardia episodes, short PR interval and bundle branch block in young healthy persons with normal heart structure. ${ }^{1}$ Typical electrocardiographic (ECG) finding of WPW include short PR interval $(<120 \mathrm{msn})$, wide QRS complex $(>120 \mathrm{msn})$ and delta wave. When sympathetic nervous system is stimulated, life threatening tachyarrhythmia such as paroxysmal supraventricular tachycardia (PSVT), atrial fibrillation, and ventricular fibrillation. ${ }^{2}$

\section{Case}

A 25years old pregnant women with G2P1 and 38weeks of gestation was taken to the operation for elective cesarean section due to previous history of cesarean. Preanesthetic assessment of the patient which was performed preoperatively in the anesthesiology outpatient clinic revealed that she had been diagnosed with WPW and an attempt for ablation had failed at that time. In her anamnesis, the patient had no history of tachycardia, angina and syncope, and she was not receiving any therapy. Cardiology consultation was ordered. In the consultation, delta waves were observed on electrocardiography of the patient with asymptomatic WPW syndrome (Figure 1). Echocardiography was normal. She had a smoking history of 3packyear. No pathology was detected in the other system examinations. Family history was unremarkable. There was a history of previous cesarean section. Because of the advantages of spinal anesthesia including less stimulation of sympathetic system, and absence of multiple drug usage, the decision was made to perform cesarean section under spinal anesthesia after the patient was informed about the technique and her written consent was received.

Following intravenous cannulation, routine monitoring was made through pulse oximetry, ECG (DII), and noninvasive blood pressure arterial measurement. Antiarrhythmic drugs and defibrillator were kept available against arrhythmia risk. The patient was positioned and spinal anesthesia was carried out with $10 \mathrm{mg} 0.5 \%$ bupivacaine and $20 \mu \mathrm{cg}$ fentanyl delivered with $26 \mathrm{G}$ pencil point needle through L3L4 space. Oxygen was given in a rate of $4 \mathrm{~L} / \mathrm{min}$ with a face mask. At the 5minutes of the operation, block level was evaluated as T6, blood pressure (BP) as $115 / 62 \mathrm{mmHg}$, heart rate (HR) as 68beats/ min, and peripheral oxygen saturation $\left(\mathrm{SpO}_{2}\right)$ as $99 \%$. Sedation was not performed. Oxytocin of 20IU was infused after the delivery and placenta expulsion. Upon the patient was agitated at the $30^{\text {th }}$ minute of the operation, sedation was induced with $1 \mathrm{mg}$ midazolam and $50 \mathrm{mg}$ propofol. The operation lasted 60 minutes. The patient was hemodynamically stable during perioperative period. Postoperative block level was found as T8, BP as $121 / 65 \mathrm{mmHg}$, HR as 70beats/ min, and the patient was transferred to the primary intensive care unit for follow-up. Following ECG monitoring, patient was stable and taken to the ward, and was discharged to home on the $3^{\text {rd }}$ day of the operation.

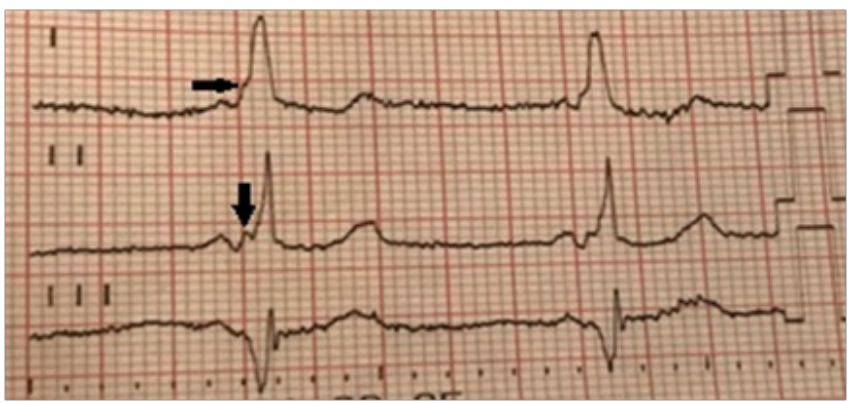

Figure I ECG of patient showing delta waves (black arrow).

\section{Discussion}

Wolff-Parkinson-White conduction is the first anatomically and echocardiographically defined preexcitation syndrome. Typical electrocardiographic (ECG) finding of WPW include short PR interval $(<120 \mathrm{msn})$, wide QRS complex $(>120 \mathrm{msn})$ and delta wave. When sympathetic nervous system is stimulated, life threatening tachyarrhythmias such as paroxysmal supraventricular tachycardia (PSVT), atrial fibrillation, and ventricular fibrillation. ${ }^{2}$ 
To main electrophysiological findings indicating the risk for sudden death are short PR interval $(<250 \mathrm{msn})$ and accessory pathway $(<270 \mathrm{msn}){ }^{3}$ Treatment is not necessary in asymptomatic patients, and the risk for development of perioperative paroxysmal supraventricular tachycardia (PSVT) is low. ${ }^{4}$ The main goal of anesthesia induction in patients with WPW syndrome is to maintain hemodynamics, and to avoid the factors that may lead to the activation of sympathetic nervous system including hypoxia, ischemia, electrolyte imbalance, surface anesthesia, mechanical stress, and stress response to intubation. ${ }^{5}$ It has been shown that fentanyl and midazolam are not effective on the accessory pathway where general anesthesia is preferred. ${ }^{6}$ It has been reported that propofol was not as effective as thiopental on the refractory period of the accessory pathway, and return to sinus rhythm was seen in patients with WPW syndrome, after propofol administration. Atropine and ketamine should be avoided, because these agents triggers tachycardia. ${ }^{7}$ It has been reported that among the inhaler agents, isoflurane and sevoflurane have no effect on the accessory pathway. Rocuronium and vecuronium are preferable muscle relaxants because of their stabilizing effect on the cardiovascular system. ${ }^{8,9}$

Regional anesthesia can be preferred in these cases, because of it prevents the use of multiple drugs, and the risk of sympathetic activation is lower with this method. ${ }^{3}$ Recent studies have shown that the use of regional anesthesia technique may be beneficial in preventing sympathetic activation. ${ }^{3,10}$ The number of cases including spinal anesthesia administration in patients with WPW syndrome is insufficient to draw a definitive conclusion. In some studies, a combination of low-dose bupivacaine and opioids have been used in patients with anesthesia induction using low-dose bupivacaine. In the case of level elevation in spinal block, blocking of the cardiovascular fibers and suppression of normal AV conduction may be encountered. ${ }^{11}$ In addition, relative activity of the parasympathetic nervous system can facilitate the accessory conduction. The dose of local anesthetic which will be delivered during spinal anesthesia should be carefully titrated at a minimum level required for the surgery. ${ }^{11}$

Phenylephrine is preferable in the treatment of hypotension in patients with WPW syndrome, because it avoids hypotension without increasing the cardiac rhythm. Fluid loading can be attempted in order to prevent a decrease in atrial filling, which causes arrhythmogenic.

\section{Conclusion}

In this case, we preferred spinal anesthesia method in order to prevent tachyarrhythmia that may occur in the pregnant woman during cesarean section. However, further randomized studies in a larger population are needed in order to make a definite judgement.

\section{Acknowledgements}

None.

\section{Conflict of interest}

The author declares there is no conflict of interest

\section{References}

1. Wolff L, Parkinson J, White PD. Bundle-branch block with short PR interval in healthy young people prone to paroxysmal tachycardia. $A m$ Heart J. 1930;5(6):685-704.

2. Kadoya T, Seto A, Aoyama K, Takenaka I. Development of rapid atrial fibrillation with a Wide QRS complex after neostigmine in a patient with intermittent Wolff-Parkinson- White syndrome. $\mathrm{Br} J$ Anaesth. 1999;83(5):815-818.

3. Kaur S, Gupta P, Aggarwal S. Anaesthetic management of WolffParkinson-White syndrome for elective caesarean section. Int $J$ Pharm Pharm Sci. 2012;4:755-756.

4. Shora A, Gurkoo S, Farooqi A, et al. Anaesthetic management of Wolff Parkinson White syndrome for caesarean section. The Internet Journal of Anaesthesiology. 2007;16:2.

5. Günüșen İ, Karaman S, Töz Z, et al. Suddenly developed atrial fibrillation during caesarean section: Wolff-Parkinson-White syndrome. Turkiye Klinikleri. J Anest Reanim. 2007;5(3):158-160.

6. Lubarsky D, Kaufman B, Turndorf H. Anesthesia unmasking benign Wolff-Parkinson-White syndrome. Anesth Analg. 1989;68(2):172-174.

7. Kabade SD, Sheikh S, Periyadka B. Anaesthetic management of a case of Wolff-Parkinson-White syndrome. Indian J Anaesth. 2011;55(4):381-383.

8. Rahul S, Patel RD, Dewoolka. Anesthetic management of WPW syndrome. The Internet Journal of Anesthesiology. 2006;11(2):1-5.

9. Chhabra A, Trikha A, Sharma N. Unmasking of benign Wolff-ParkinsonWhite pattern under general anesthesia. Indian J Anesth. 2003;47:208-211.

10. Sahu S, Karna ST, Karna A, et al. Anaesthetic management of WolffParkinson-White syndrome for hysterectomy. Indian $J$ Anaesth. 2011;55:378-380.

11. Garg R, Sinha R, Nishad PK. Patient with Wolff-Parkinson-White syndrome with intermittent pre-excitation under subarachnoid block for urological surgery. Indian J Anaesth. 2011;55(2):167-170. 